\title{
Determination of Local Oxidations States in Ni-NiO Core-shell Structures Using White Line Intensity Ratios
}

\author{
S.M. Weil*, J. Wang**, J.B. Tracy**, and K. van Benthem* \\ * Department for Chemical Engineering and Materials Science, University of California, Davis, 1 \\ Shields Ave., Davis CA 95616 \\ ** Materials Science \& Engineering Department, North Carolina State University, Raleigh NC
}

During the oxidation of nickel nanoparticles at elevated temperatures, the inwards diffusion of anions is slower than outwards diffusion of metal cations. This results in a net flux of vacancies into the volume of the nanoparticles, which is commonly referred to as the nanoscale Kirkendall effect [1]. Upon super-saturation and coalescence of the vacancies, partially hollow core-shell structures form with a shell of $\mathrm{NiO}$ and a remaining core of metallic nickel [2].

Here, we report the atomic-scale characterization of nickel oxidation as determined by electron energy-loss spectroscopy (EELS) in the aberration-corrected scanning transmission electron microscope (STEM). Figure 1a shows a high-angle annular dark-field image of a partially oxidized nickel nanoparticle with a diameter of roughly $27 \mathrm{~nm}$. Spectrum imaging was used to obtain spatially resolved EELS data of the $\mathrm{O} K$ and $\mathrm{Ni} \mathrm{L}_{2,3}$ absorption edges (see Figure 1b). The integrated intensity ratio of $\mathrm{L}_{3}$ and $\mathrm{L}_{2}$ ionization edges of $3 \mathrm{~d}$ transition metals in electron energy-loss spectroscopy gives quantitative information about the local valence band occupancy of the metal atoms, and, hence, their oxidation state [3].

A new technique for the determination of the so-called white line intensity ratios is proposed. Our method uses smoothing of the spectra by least squares fitting of a $4^{\text {th }}$-order polynomial function in the form of piecewise splines. Free knots and knot removal techniques were used to handle the noise of the spectra by manipulating the degrees of freedom of the spline while maintaining an accurate fit. The $\mathrm{L}_{3}$ and $\mathrm{L}_{2}$ ionization edges are identified through the local maxima of the fitted data. Linear functions were used to connect the inflection points on either side the peaks. The intensities of the "spline-fitted spectra" above the linear intersects were then integrated between the inflection points (see Figure 2). The proposed technique for the determination of $\mathrm{L}_{3} / \mathrm{L}_{2}$ intensity ratios does not rely on accurate background fitting and modeling of inelastic scattering cross-sections to distinguish contributions from unoccupied states with $s$ and $d$ symmetry. The reliability of this method will be discussed in comparison to established routines from the literature [3].

[1] Y.D. Yin, R.M. Rioux, C.K. Erdonmez, S. Hughes, G.A. Somorjai, A.P. Alivisatos, Science 304 (2004) 711-714.

[2] J.G. Railsback, A.C. Johnston-Peck, J. Wang, J.B. Tracy, Size-Dependent Nanoscale Kirkendall Effect During the Oxidation of Nickel Nanoparticles (submitted for publication)

[3] R.D. Leapman et al., Phys. Rev. B 26 (1982) 614-635

[4] KvB acknowledges financial support through start-up funds from the University of California at Davis. Parts of this research were carried out at the National Center for Electron Microscopy (NCEM) at Lawrence Berkeley National Laboratory. Technical assistance by Quentin Ramasse is greatly appreciated. 

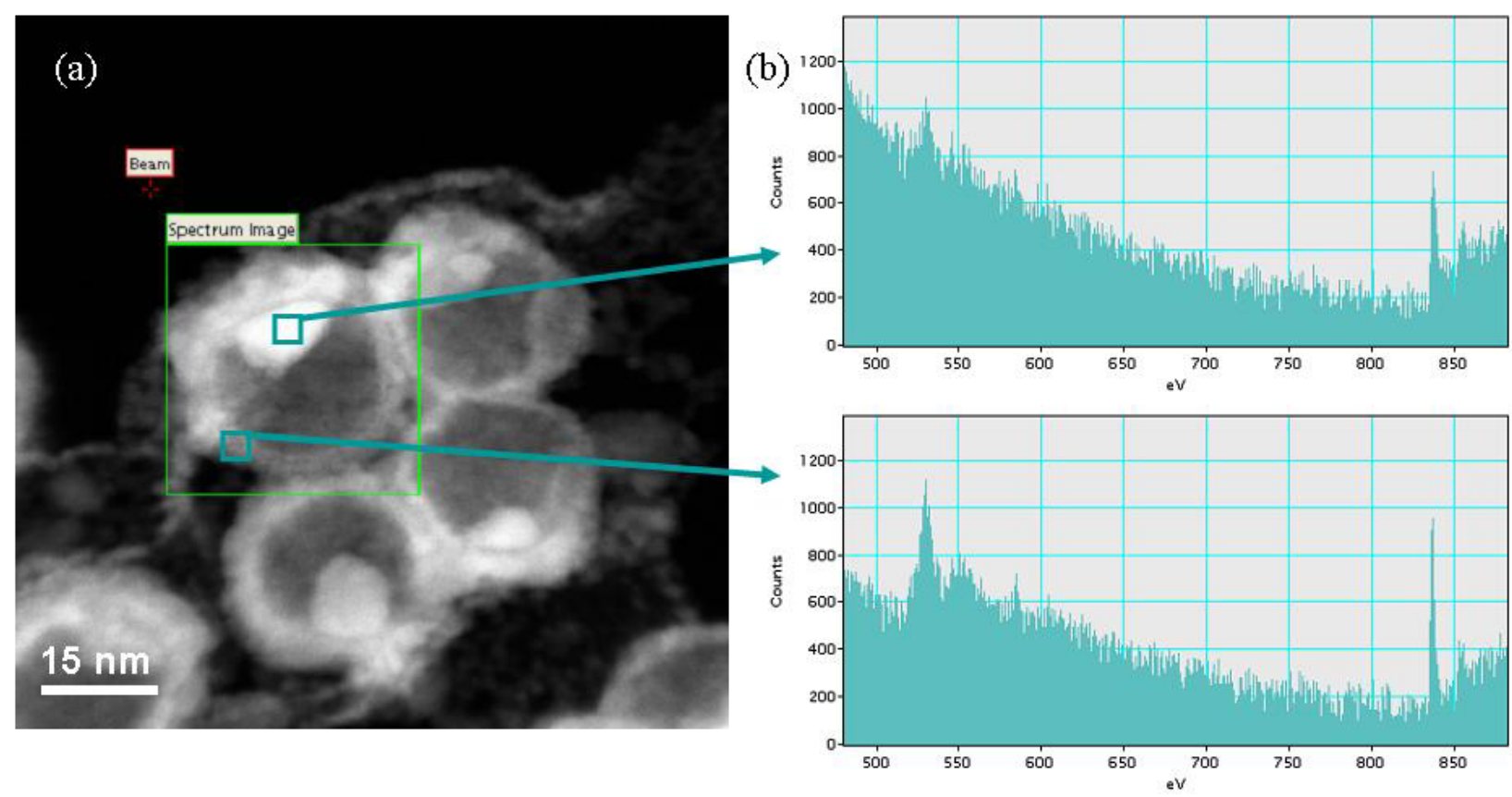

Figure 1. HAADF STM image of Ni-NiO core-shell particles and two EELS spectra extracted from a corresponding spectrum image. The energy-loss axis was not calibrated.

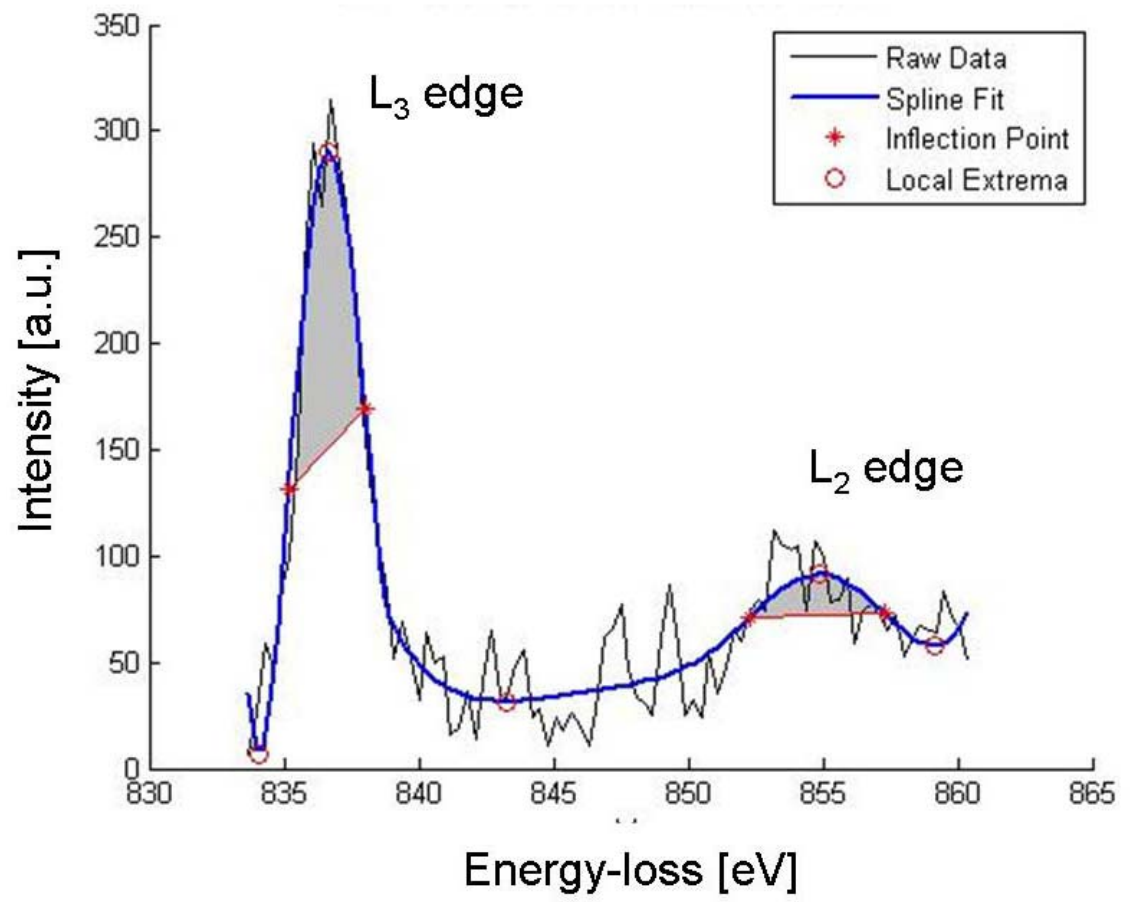

Figure 2. Single $\mathrm{Ni}_{2,3}$ spectrum extracted from a spectrum image (raw data and splined fit). The shaded areas represent the integration windows for the determination of $\mathrm{L}_{3} / \mathrm{L}_{2}$ intensity ratios. The energy-loss axis was not calibrated. 